Revisión de la literatura 


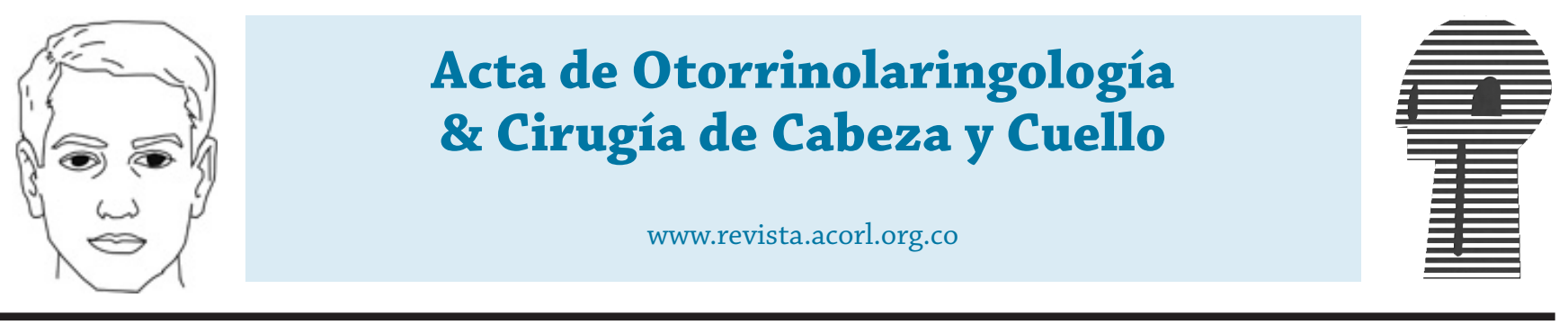

\title{
Artroscopia diagnóstica en cirugía maxilofacial
}

\section{Diagnostic arthroscopy in Maxillofacial Surgery}

\author{
José Manuel Valdés-Reyes*, Galo Fernando Guzmán-Castillo**, Paula Melissa Pardo-Chirivi***, María Teresa
} Russi-Santamaría****, Arnulfo Andrade-Castro ${ }^{* * * * *}$.

\footnotetext{
* Departamento de Cirugía Oral y Maxilofacial. Pontificia Universidad Javeriana. ORCID: https://orcid.org/0000-0003-0297-0716

** Departamento de Cirugía Oral y Maxilofacial. Pontificia Universidad Javeriana. email: galofernandogc@gmail.com ORCID: https:// orcid.org/0000-0002-3368-2963

*** Departamento de Cirugía Oral y Maxilofacial. Pontificia Universidad Javeriana. email: 62.chirivi.paula@gmail.com ORCID: https:// orcid.org/0000-0002-4219-382X

**** Departamento de Cirugía Oral y Maxilofacial. Profesor de la Pontificia Universidad Javeriana. Colombia. Cirujano Maxilofacial Hospital Universitario Méderi, Bogotá Colombia. email:materussi@hotmail.com ORCID: https://orcid.org/0000-0001-6933-3995

***** Departamento de Cirugía Oral y Maxilofacial. Profesor de la Pontificia Universidad Javeriana. Colombia. Cirujano Maxilofacial Hospital Universitario Méderi, Bogotá Colombia. email:arancas@gmail.com
}

Forma de citar: Valdés-Reyes JM, Guzmán-Castillo GF, Pardo-Chirivi PM, Russi-Santamaría MT, Andrade-Castro A. Artroscopia diagnóstica en cirugía maxilofacial. Acta otorrinolaringol. cir. cabeza cuello. 2021;49(2):148-155. DOI.10.37076/acorl.v49i2.525

\section{INFORMACIÓN DEL ARTÍCULO}

Historia del artículo:

Recibido: 3 de Mayo de 2020

Evaluado: 24 de Mayo de 2021

Aceptado: 15 de Junio de 2021

Palabras clave (DeCS):

Artroscopía, articulación temporomandibular, cirugía bucal.

\section{RESUMEN}

Introducción: los desarreglos internos de la articulación temporomandibular (ATM) son los más comunes. La artroscopia es de gran utilidad en cirugía maxilofacial como alternativa para el diagnóstico y el tratamiento de los desarreglos internos de la ATM. El objetivo de la presente revisión es describir la artroscopia diagnóstica de la ATM. Métodos: se realizó una revisión narrativa de la literatura y una búsqueda en las bases de datos PubMed, ProQuest, SciELO, Mendeley y Elsevier empleando los descriptores (artroscopia diagnóstica, articulación temporomandibular) en español e inglés. Se seleccionaron los artículos publicados en un período de 40 años (19802020), incluyendo en el estudio un total de 26 artículos y 6 libros de 702 documentos revisados. Discusión: en la artroscopia de la ATM se puede observar la posición, calidad y textura del cartílago articular, la vascularización y la redundancia de la membrana sinovial y se pueden realizar procedimientos como técnicas de artros- 
copia avanzada. Conclusiones: la artroscopia diagnóstica facilita el diagnóstico y manejo de las patologías intraarticulares, por lo que el conocimiento de esta técnica resulta imprescindible.

\section{ABSTRACT}

Key words (MeSH):

Artroscopía, articulación

temporomandibular, cirugía bucal.
Introduction: Internal disorders of the TMJ are the most common. Arthroscopy is very useful in Maxillofacial Surgery as an alternative for the diagnosis and treatment of internal disorders of the Temporomandibular Joint. The aim of this review is to describe the diagnostic arthroscopy of the Temporomandibular Joint. Methods: A narrative literature review and search of PubMed, ProQuest, SciELO, Mendeley and Elsevier databases were performed in English and Spanish using the descriptors (Diagnostic arthroscopy, Temporomandibular Joint) in Spanish and English. Articles published over a period of 40 years (1980-2020) were selected, including a total of 26 articles and 6 books from 702 reviewed documents in the study. Discussion: In the arthroscopy of the Temporomandibular Joint the position, quality, texture of the articular cartilage, the vascularization, and the redundancy of the synovial membrane can be observed, and procedures such as advanced arthroscopic techniques can be performed. Conclusions: Diagnostic arthroscopy facilitates the diagnosis and management of intra-articular pathologies. Being necessary and essential knowledge of this technique.

\section{Introducción}

Los desórdenes más comunes de la articulación temporomandibular (ATM) son los desarreglos internos. Las opciones de tratamiento para los trastornos temporomandibulares han mejorado significativamente desde la llegada de la artroscopia $(1,2)$.

Se han descrito diferentes alternativas para tratar estas entidades, como procedimientos conservadores con férulas, fármacos, fisioterapia o procedimientos quirúrgicos mínimamente invasivos, como la artrocentesis, artroscopia y cirugía abierta como última instancia. La utilidad de la artroscopia en especialidades como ortopedia y cirugía maxilofacial se ha vuelto de gran interés gracias a las ventajas que ofrece en el diagnóstico y tratamiento de la ATM $(1,3)$.

La capacidad de tener un examen directo de las articulaciones en el que se puede observar de forma clara y precisa la posición, calidad, textura del cartílago articular, la vascularización y la redundancia de la membrana sinovial han convertido a la artroscopia en una técnica muy utilizada por muchos cirujanos. Esta tiene como objetivo principal realizar una lisis y la eliminación de las adherencias que se localizan entre las superficies articulares; se realiza principalmente bajo anestesia general y es dependiente del equipo más la habilidad del cirujano $(1,4)$.

Los primeros exámenes de artroscopia se remontan a 1918 cuando Tagaki utilizó un cistoscopio para observar la rodilla de un cadáver. La primera artroscopia diagnóstica en la ATM fue realizada en 1974 por Masatoshi Ohnishi, en Japón, utilizando el artroscópico N. ${ }^{\circ} 24$ desarrollado por Watanabe $(5,6)$.

El principal uso quirúrgico de la artroscopia se convirtió en lisis y lavado popularizado por Sanders, quien utilizó por primera vez el término lisis en 1986. El tamaño reducido de la ATM fue la razón por la que la artroscopia operatoria se veía limitada; para esto, se diseñaron los instrumentos en miniatura y, posteriormente, el doctor McCain desarrolló técnicas de sutura con artroscopia operatoria $(1,3)$.

Los trastornos temporomandibulares se definen como los tipos de alteraciones que afectan al componente articular o extraarticular. Dentro de los desórdenes intraarticulares o denominados intracapsulares se encuentra la alteración discal con o sin reducción, adherencia discal o adhesión discal, subluxación temporomandibular y luxación articular $(6,7)$.

La ATM está compuesta por superficies articulares, tanto del temporal (cavidad glenoidea y eminencia articular) como también el cóndilo mandibular, disco articular, cápsula revestida de membrana sinovial, medios de unión (sus ligamentos) y estructuras accesorias (los músculos masticadores). Siempre se debe tener en cuenta estructuras anatómicas pericapsulares como la arteria y la vena temporal superficial, la arteria y la vena facial transversa, ramas de la vena y la arteria temporal superficial y estructuras nerviosas como el nervio auriculotemporal. Entre las superficies articulares y el disco se forman dos compartimentos: uno superior localizado entre el disco articular y las superficies articulares del hueso temporal; y otro inferior entre el disco articular y el cóndilo mandibular, limitado por delante y por detrás por el ligamento capsular $(1,8)$.

Es de gran interés comprender el volumen de líquido sinovial en los compartimientos superior e inferior de la articulación. En el espacio articular superior el volumen aproximado es de $2 \mathrm{~mL}$, que puede aumentar hasta $6 \mathrm{~mL}$ en condiciones patológicas, a diferencia del espacio articular inferior, que aproximadamente contiene $1 \mathrm{~mL}$ de líquido sinovial y también en condiciones patológicas puede aumen- 
tar a $2 \mathrm{~mL}$. Esto es esencial para prevenir desgarros de la cápsula cuando se intenta realizar la punción inicial y la insuflación de la articulación (9).

Por ser una articulación denominada gínglimoartrodial también es importante saber que el compartimento superior es el que está involucrado en el movimiento de traslación o deslizante, y es aquí en donde ocurre la mayoría de las alteraciones.

Con la artroscopia diagnóstica podemos visualizar directamente las alteraciones internas de la ATM, lo cual no es posible con otros estudios de imagen aunque sean muy avanzados; por esta razón, se hace necesario el conocimiento de este tema. La motivación principal es encontrar los diagnósticos reflejados en la literatura que se pueden obtener con la artroscopia diagnóstica de la ATM; por tanto, el objetivo de la presente revisión es describir la artroscopia diagnóstica de la ATM.

\section{Metodología}

Se realizó una búsqueda bibliográfica en las bases de datos globales PubMed, ProQuest, SciELO, Mendeley y Elsevier, en idioma inglés y español, en las que se seleccionaron inicialmente 100 artículos publicados en un período de 40 años (1980-2020), en categorías de investigación y revisión, empleando los descriptores: "artroscopia diagnóstica", "articulación temporomandibular", "Diagnostic Arthroscopy" y "Temporomandibular Joint". Se encontraron 702 artículos. Estos se revisaron por sus títulos y contenido y se ordenaron por relevancia. Seguidamente, se excluyeron los artículos que repetían información y no aportaban información nueva o actualizada. Se tuvieron en cuenta artículos que hicieran referencia a indicaciones, complicaciones, técnica quirúrgica y conceptos actuales sobre artroscopia. Para el análisis final de esta revisión se tuvieron en cuenta solo 26 artículos y 6 libros.

\section{Discusión}

Es imperativo que se tenga presente la anatomía de la región específica en una cirugía artroscópica. En las fosas glenoideas delgadas es posible que un trocar afilado perfore la delgada capa ósea. La proximidad al conducto auditivo externo, vasos temporales y el nervio facial también son motivo de gran preocupación durante la maniobra de punción (10).

Las áreas que se pueden examinar en una artroscopia de ATM (Figura 1) en el espacio articular superior en el siguiente orden son: cubierta sinovial medial, sombra pterigoidea, sinovial retrodiscal, vertiente posterior de eminencia articular y fosa glenoidea, disco articular, zona intermedia y receso anterior (1).

\section{Tipos de artroscopia}

Realizar una historia clínica y examen clínico de manera completa y cuidadosa es de gran importancia debido a que ninguna técnica suplementaria puede suplir un examen clínico completo. Es por esto por lo que, con la ayuda de las imágenes diagnósticas como la tomografía axial computa-

A

B

C

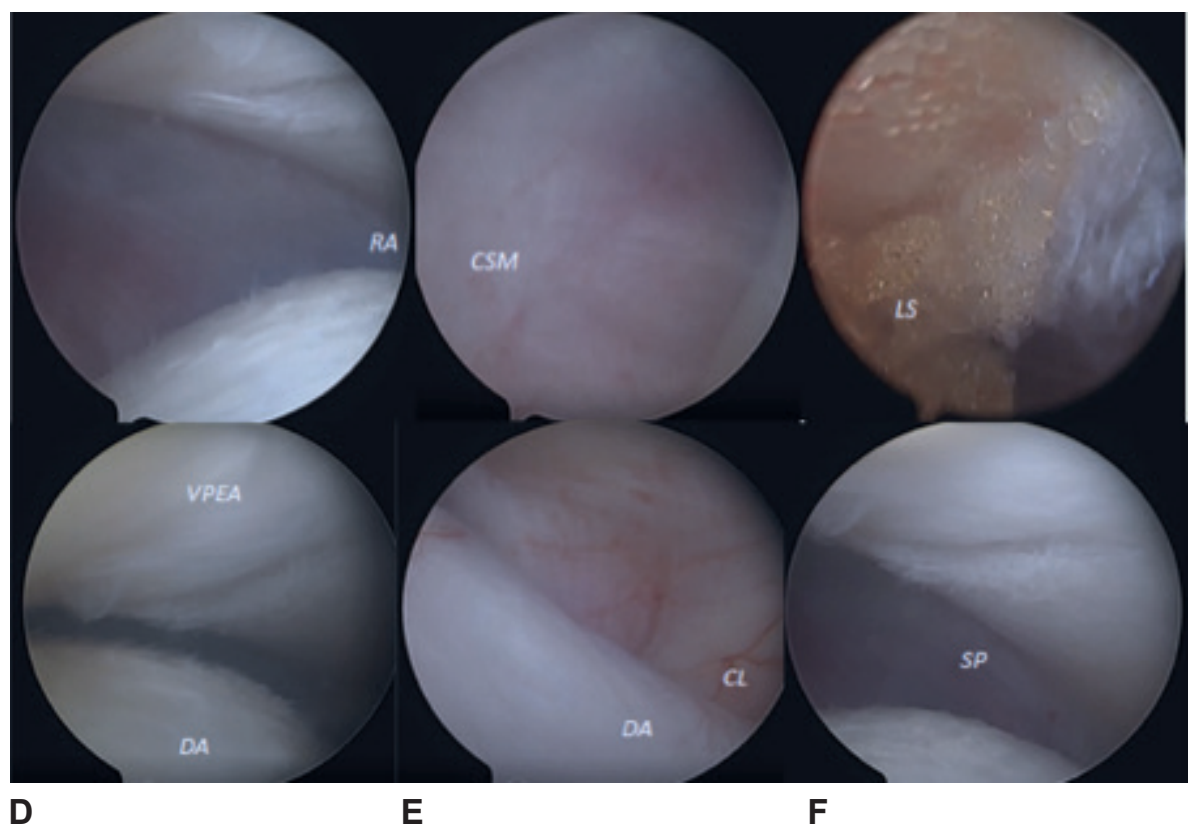

Figura 1. Estructuras anatómicas que se observan en la artroscopia. A. Receso anterior (RA); B. Cubierta sinovial medial (CSM); C. Espacio articular inferior, donde se observa líquido sinovial (LS); D. Vertiente posterior de eminencia articular (VPEA) y disco articular (DA); E. Cápsula lateral (CL) y disco articular (DA); F. Sombra pterigoidea (SP).

Fuente: imágenes tomadas por los autores de la revisión. 
rizada (TAC) y la resonancia magnética nuclear (RMN), es posible realizar un correcto diagnóstico y tratamiento. Es aquí en donde la artroscopia aporta gran información como un método de ayuda diagnóstica y también de tratamiento; los tipos de artroscopias son: diagnóstica y avanzada, esta última se encarga del tratamiento quirúrgico de la articulación (11).

\section{Indicaciones}

La artroscopia está indicada para trastornos internos de la ATM, alteraciones discales, osteoartritis, enfermedades reumáticas, pseudotumores sinoviales, bloqueo cerrado, osteoartrosis, condromatosis sinovial, hipermovilidad con bloqueo abierto y luxación recurrente (11-13).

\section{Contraindicaciones}

Las contraindicaciones absolutas son anquilosis ósea, reabsorción avanzada de la fosa glenoidea, infección y tumores malignos $(11,14)$. Las contraindicaciones relativas son un paciente con alto riesgo de hemorragia, un paciente con alto riesgo de infección y anquilosis fibrosa $(11,14)$.

\section{Técnica de artroscopia}

La artroscopia por punción simple o primaria inicia con la preparación del paciente en la sala de operaciones colocado en posición supino-dorsal; la cabeza debe girarse a un lado para que mantenga un nivel plano y se debe realizar una preparación de la piel preauricular (se coloca la mecha en el oído y se realiza el marcaje para la punción). Posteriormente, se infiltra solución anestésica, preferiblemente sin vasoconstrictor a nivel preauricular y en espacio articular superior, lo cual permite la insuflación del espacio articular superior por medio de un abordaje inferior y lateral utilizando lidocaína aproximadamente de $2,5 \mathrm{cc}$.

Después se realiza la punción; primero, una incisión con hoja \#11 a nivel preauricular según el abordaje a realizar, siendo el posterosuperior el más común; después, la primera punción siempre se coloca en la máxima concavidad de la fosa glenoidea con la mandíbula protruida. Luego se procede a la inserción de la aguja de salida, con la mandíbula protruída. El asistente quirúrgico insufla la articulación $2 \mathrm{~mL}$ a $3 \mathrm{~mL}$ de líquido y el cirujano inserta un calibre 22 y de una pulgada y media, aproximadamente $5 \mathrm{~mm}$ anterior e inferior del sitio de punción de la fosa. Se continúa con la artrocentesis artroscópica con 120 cc de irrigación con turbulencia para eliminar restos inflamatorios y lisis de adherencias; incluso, el mismo barrido del artroscópico ayuda a liberar las adherencias. También se puede aplicar una inyección de agentes esclerosantes en el tejido retrodiscal, una inyección de esteroides, una inyección de ácido hialurónico, plasma rico en plaquetas, células madre, entre otros.

Al finalizar, se procede con el retiro de todos los instrumentos manteniendo una presión directa sobre los sitios de punción. Se debe elevar la cabeza del paciente para ayudar en la hemostasia, realizar una sutura única 6-0 nylon y se debe retirar la mecha del oído. También se han descrito modificaciones de la técnica, como es el caso de la artroscopia avanzada con un solo puerto de entrada para el procedimiento e irrigación $(5,10,15,16)$.

Después se realiza la manipulación de la mandíbula para verificar los rangos de movimiento; además, recomendamos una laringoscopia directa para verificar la permeabilidad de la vía aérea.

Otra técnica es la artroscopia por punción doble u operativa, que consiste en la técnica antes descrita, pero se realiza una segunda punción en la esquina más anterior y lateral del espacio articular superior para garantizar flexibilidad de la cánula operatoria, y así poder realizar diferentes procedimientos como biopsias, desbridamientos, meniscopexia, entre otros (10).

\section{Abordajes en artroscopia}

Existen cinco abordajes clásicos (Figura 2) para realizar la artroscopia en la ATM (17):

\section{Abordajes para la artroscopia de la ATM}
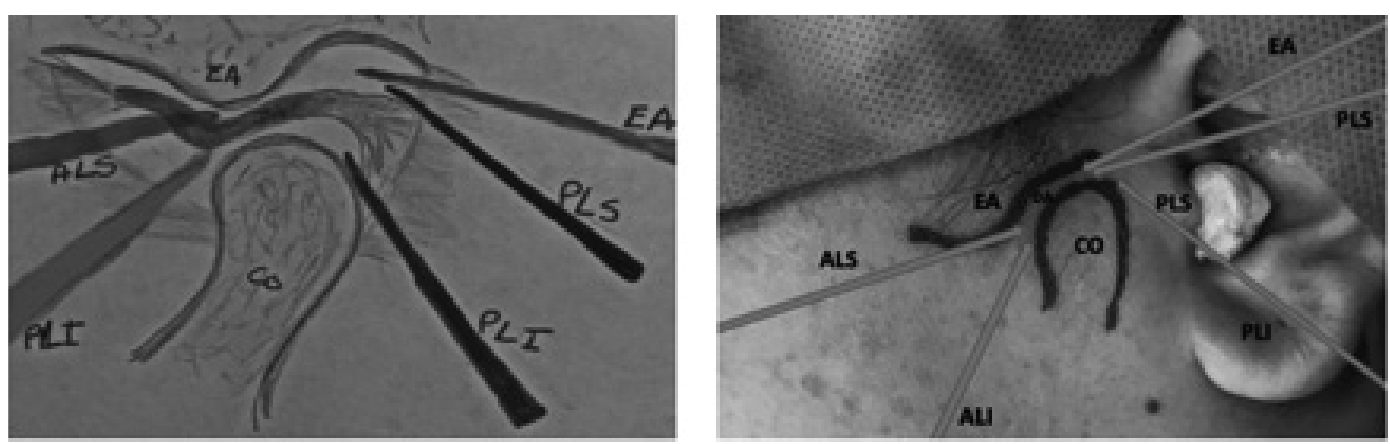

Derechos de autor: Imágenes tomadas por autores de la revisión.

Figura 2. Diagrama de los tipos de abordajes para la artroscopia de la ATM. ALI (anterolateral infeiror); ALS (anterolateral superior); Co (cóndilo); DA (disco articular); EA (endaural); EA (eminencia articular); PLI (posterolateral inferior ); PLS (posterolateral superior o Murakami).

Fuente: imágenes tomadas por los autores de la revisión. (si es endaural) 
1. posterolateral superior o abordaje de Murakami;

2. anterolateral superior;

3. posterolateral inferior;

4. anterolateral inferior;

5. y endaural.

\section{Instrumental}

El principal instrumento es el artroscópico: está compuesto por un lente/fibra óptica, una fuente de luz, una cámara y un monitor. En el sistema artroscópico ideal para ATM se debe analizar la angulación del lente (se recomienda 30 grados), la resolución, la distancia focal y el tipo de fibra óptica. Para la artroscopia operatoria o terapéutica es necesario: fórceps, tijeras, sondas, graspers, bisturí, raspadores, curetas e instrumentos motorizados. El artroscópico se puede conectar a una torre para la visualización en un monitor, la grabación y la fuente de luz (10).

\section{Hallazgos en la artroscopia diagnóstica}

\section{Adherencias}

Las adherencias (Figura 3) son bandas de fibrosis que se forman dentro de la articulación, que pueden alterar la movilidad articular, lo que contribuye al dolor y la disfunción de la ATM. La entidad patológica denoninada como adherencia fibrosa fue descrita por Murakami y colaboradores (18) en 1986, aunque Kaminishi y Davis (19) en 1989 demostraron varios tipos de adhesiones.
De acuerdo con Kaminishi y Davis (19), estas se clasifican en:
A. Banda fibrosa simple
B. Banda fibrosinovial
C. Banda discal ósea
D. Falsa pared o pseudopared.

\section{Sinovitis}

La sinovitis es la inflamación de la membrana sinovial (Figura 4), que usualmente se manifiesta con dolor en la ATM. Esta se caracteriza por hiperemias, angiogénesis, edemas y proliferación capilar (18-21).

La clasificación de la sinovitis basada en la artroscopia de Holmlund y Hellsing (22) es:

- Sinovitis 0: revestimiento sinovial normal, pálido, casi translúcido, con una fina red de pequeños vasos sanguíneos anastomosados.

- Sinovitis I: área localizada con mayor vascularización e hiperemia capilar. El sangrado por contacto puede ocurrir durante la artroscopia.

- Sinovitis II: hiperemia capilar generalizada, derrame y residuos. El examen artroscópico es posible después de la irrigación de la cavidad articular.

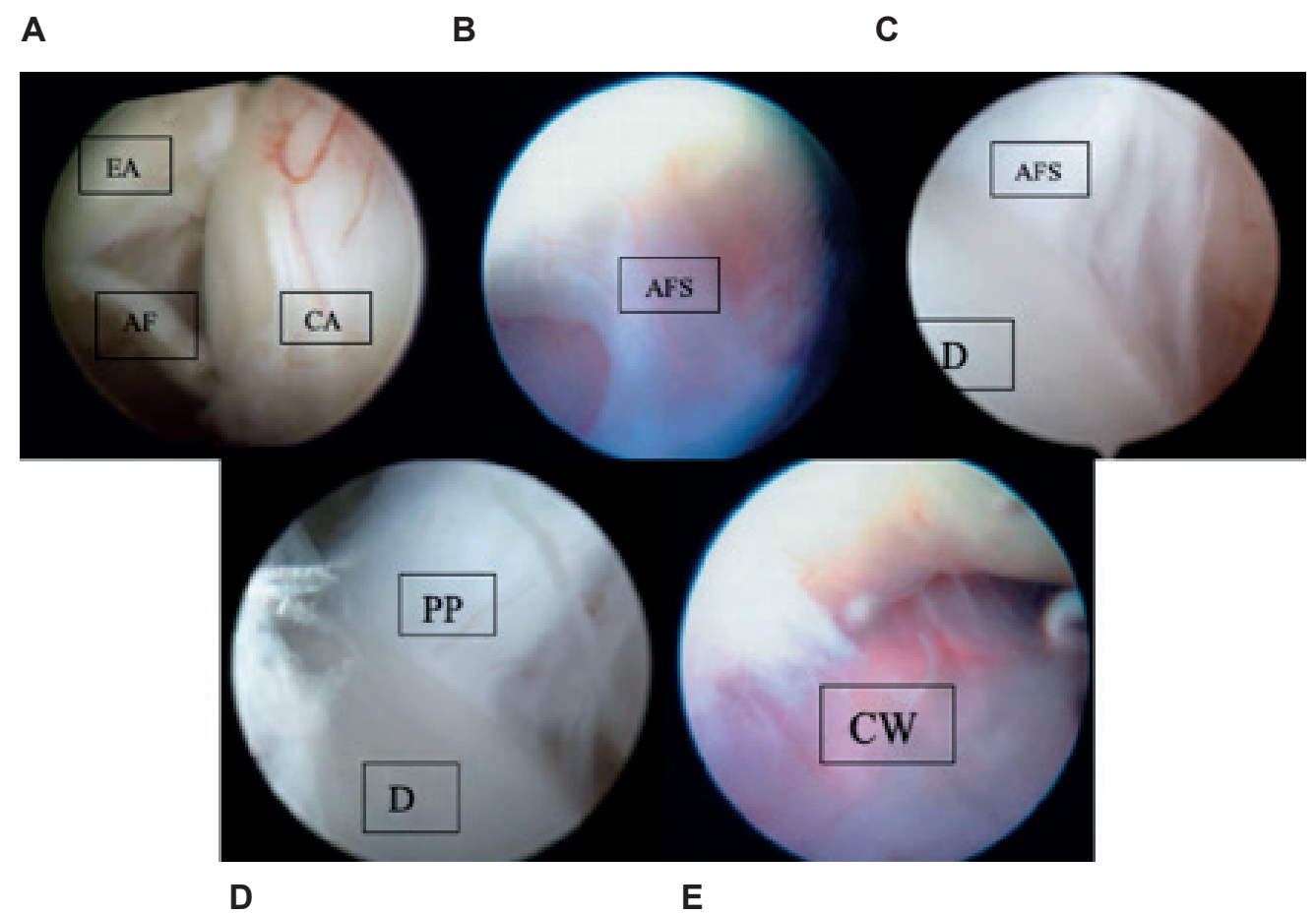

Figura 3. Tipos de adherencias en la ATM. A. AF: adherencia fibrosa; CA: cóndilo articular; EA: eminencia articular. B. AFS: adherencia fibrosinovial. C. AFS: adherencia fibrosinovial; D: disco articular. D. D: disco articular; PP: pseudopared. E. CW: cobweb.

Fuente: imágenes tomadas por los autores de la revisión. 
A

B

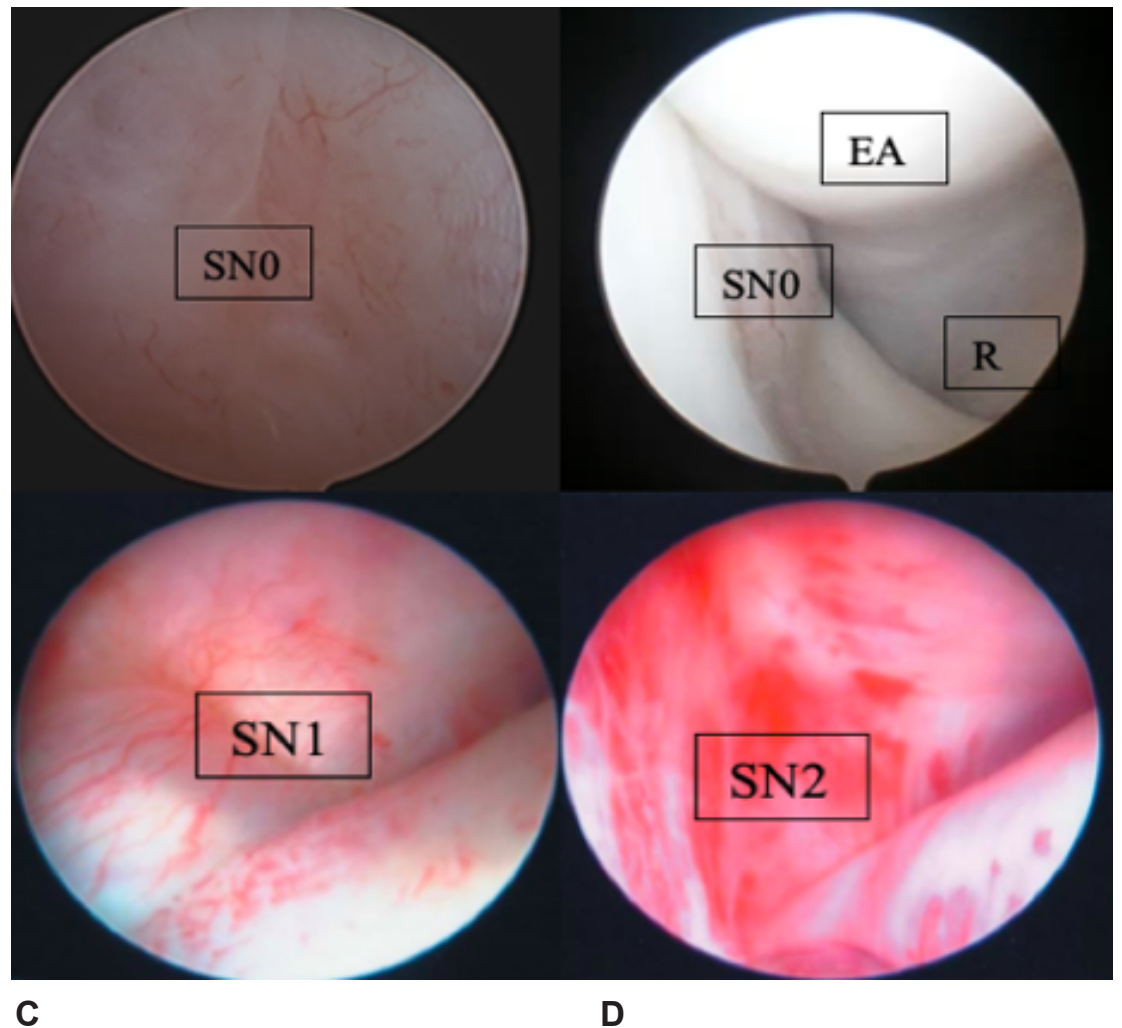

Figura 4: Tipos de sinovitis en la ATM. A. SNO: sinovitis grado 0; B. EA: eminencia articular, R: receso anterior, SNO: sinovitis grado 0; C. SN1: sinovitis grado I; D. SN2: sinovitis grado II.

Fuente: imágenes tomadas por los autores de la revisión.

\section{Cambios degenerativos}

La artrosis u osteoartrosis es un desorden degenerativo que se caracteriza por tres fenómenos: la destrucción del cartílago de la superficie articular, la remodelación ósea con fenómenos de neoformación (osteofitos) y de rarefacción ósea (quistes subcondrales), y la sinovitis secundaria (22).

La clasificación de la osteoartrosis o condromalacia (23) se divide en:

- Grado I (superficial): cartílago aparece edematoso y se marca la huella de la artroscopia a la palpación del cartílago.

- Grado II (intermedio): arrugas en el cartílago como consecuencia de la rotura del colágeno.

- Grado III (intermedio avanzado): fibrilación y ulceración del cartílago.

- Grado IV (avanzado): exposición del hueso subcondral.

\section{Cuerpos sinoviales}

Son nódulos cartilaginosos que ocupan el espacio articular, y son hallazgos frecuentes en la condromatosis sinovial (18).

\section{Desplazamiento discal}

\section{Roofing}

En 1996, McCain se refiere al término roofing, el cual ayuda de una forma más objetiva a verificar y cuantificar el desplazamiento discal por medio de la evaluación de la ubicación de la unión posterior del disco con el tejido retrodiscal; si esta se ubica o se visualiza en la vertiente posterior de la eminencia articular del cóndilo, se habla de roofing del $100 \%$ (ubicación adecuada del disco). A medida que el disco es desplazado anteriormente, disminuye el roofing; esto es proporcional a la gravedad del cuadro (19).

Correlación entre hallazgos clínicos/imagenológicos (clasificación de Wilkes) y hallazgos artroscópicos de articulación temporomandibular (2, 24-26)

Existen varias clasificaciones para los desórdenes articulares, pero la clasificación de Wilkes es la que se ha correlacionado más con hallazgos artroscópicos; correlación hecha por Bronstein y Merrill.

- Estadio I (inicial): clínicamente se detecta clic recíproco, indoloro. En la RMN se observa desplazamiento discal con reducción, y en la artroscopia se puede obser- 
var un roofing del $80 \%$ en boca cerrada y del $100 \%$ en boca abierta; además, elongación incipiente de la zona bilaminar.

- Estadio II (inicial-intermedio): clínicamente se detecta chasquido intenso y tardío en la apertura, bloqueos y algias ocasionales. En la RMN se observa desplazamiento discal con reducción, engrosamiento de la banda posterior. y en la artroscopia se puede observar un roofing del $50 \%$ en boca cerrada y del $100 \%$ en boca abierta; además, elongación de la zona bilaminar y sinovitis adhesiva.

- Estadio III (intermedio): clínicamente se detecta mayor número de episodios de bloqueo, dolor más intenso y frecuente. En la RMN se observa deformidad articular evidente, y en la artroscopia se puede observar un roofing del $5 \%$ en boca cerrada y del $15 \%$ en boca abierta, condromalacia de grados I, II o III; además, adherencias visibles y sinovitis prominente.

- Estadio IV (intermedio-avanzada): clínicamente se detecta dolor crónico variable, cefaleas, limitación del movimiento en fases irregulares. En la RMN y la TAC se evidencian cambios óseos leves-moderados, y en la artroscopia se puede observar hialinización o metaplasia de la banda posterior, condromalacia gado III o IV.

- Estadio V (avanzada): clínicamente se evidencia empeoramiento progresivo de la función y crepitaciones articulares. En la RMN se observa gran deformidad del disco y en la TAC se evidencian signos degenerativos, esclerosis subcondral, osteofitos; en la artroscopia se puede observar fibrilación o perforación discal, condromalacia grado IV, adherencias fibrosas generalizadas y falsas paredes, y sinovitis avanzada.

\section{Complicaciones de la artroscopia $(27,28)$}

- Otológicas: desgarros del conducto auditivo externo, coágulos de sangre en el canal auditivo externo, lesiones de membrana timpánica, pérdida de la audición y vértigo.

- Neurológicas: lesión del nervio auriculotemporal, paresia del facial, parálisis del facial, lesión del nervio alveolar inferior y alteraciones cardíacas (reflejo trigémino-cardíaco).

- Vascular: fístula arteriovenosa y hematoma extradural.

- Ocular: alteración de la precisión visual.

- Infección y rotura de instrumentos.

- Extravasación faríngea lateral.

- Ruptura de instrumentos.

Diferentes estudios sobre la técnica de la artroscopia diagnóstica en la ATM muestran resultados prometedores. Murakami y colaboradores (29), en un estudio donde se compara la artrocentesis, la artroscopia y los tratamientos conservadores no quirúrgicos para el bloqueo cerrado de la ATM en 63 pacientes consecutivos tratados no quirúrgicamente, 20 pacientes tratados con artrocentesis y 25 pacientes tratados con cirugía artroscópica, concluyeron que la tasa de éxito fue del 55,6\% en el grupo no tratado quirúrgicamente, del $70 \%$ en el grupo de artrocentesis y del $91 \%$ en el grupo de artroscopia. Avellá y colaboradores (30), en un estudio de 22 articulaciones con desarreglos internos de la ATM, evaluaron el dolor y la movilidad posterior a la artroscopia. Los pacientes presentaron resultados de mejoría de la sintomatología y la función de más de la mitad de los casos, por lo que se concluyó que el lavado y la lisis articular es una técnica efectiva para los trastornos temporomandibulares en estadios tempranos y tardíos de la enfermedad. Dahlström y colaboradores (31), en un estudio que evalúa cambios en la función y el dolor en 26 pacientes posterior a la artroscopia, concluyen que la lisis y el lavado del espacio articular superior de la ATM es efectivo para aliviar el dolor persistente y la limitación en la función con baja morbilidad. McCain y colaboradores (32) realizaron un estudio de artroscopia en 9 pacientes mujeres de edad promedio 40 años, donde la limitación de la apertura bucal preoperatoria promedio fue de 25 $\mathrm{mm}$; esto mejoró posterior a la artroscopia, para un promedio de $35 \mathrm{~mm}$ en un mes y de $37 \mathrm{~mm}$ en 3 meses. La escala análoga del dolor promedio preoperatorio fue de $8 / 10$, lo que significa que mejoró a 4/10 en 1 y 3 meses.

\section{Conclusiones}

La artroscopia diagnóstica nos permite evaluar hallazgos patológicos de la ATM. Esta técnica facilita al cirujano maxilofacial el diagnóstico de sinovitis, adherencias, condromalacia, perforación del disco articular, la presencia de cuerpos extraños y entidades que pueden ser de difícil diagnóstico aun cuando se tiene a disposición imágenes de diagnóstico avanzadas. Es imprescindible el conocimiento de esta técnica en la práctica de la cirugía maxilofacial.

\section{Conflicto de interés}

Los autores declaran que no tienen ningún conflicto de interés con ninguna empresa o casa comercial.

\section{REFERENCIAS}

1. González-García R, Gil-Díez Usandizaga JL, RodríguezCampo FJ. Arthroscopic anatomy and lysis and lavage of the temporomandibular joint. Atlas Oral Maxillofac Surg Clin North Am. 2011;19(2):131-44. doi: 10.1016/j.cxom.2011.05.002

2. Higuchi K, Chiba M, Sai Y, et al. Relationship between temporomandibular joint pain and magnetic resonance imaging findings in patients with temporomandibular joint disorders. Int J Oral Maxillofac Surg. 2020;49(2):230-36. doi: 10.1016/j. ijom.2019.06.028

3. McCain JP. Arthroscopy of the human temporomandibular joint. J Oral Maxillofac Surg. 1988;46(8):648-55. doi:10.1016/02782391(88)90107-3

4. Indresano TA, Park CM. Arthroscopy of the Temporomandibular Joint. En: Fonseca RJ, Powers M, Frost DE, Le B, editores. Oral and Maxillofacial Surgery. 3.a edición. [Missouri]: Elsevier; 2018. p. 892-903.

5. McCain JP, Stroia L. Arthroscopy and Arthrocentesis of the Temporomandibular Joint. En: Miloro M, Ghali GE, Larsen 
PE, Waite PD, editores. Peterson's Principles of Oral and Maxillofacial Surgery. 3.a edición. [Shelton, CT ]: People's Medical Publishing House; 2011. p. 1069-1122.

6. Fuentes Fernández R, Carter P, Muñoz S, et al. Evaluation of validity and reliability of a methodology for measuring human postural attitude and its relation to temporomandibular joint disorders. Singapore Med J. 2016;57(4):204-8. doi:10.11622/ smedj.2015159

7. Reissmann DR, Anderson GC, Heydecke G, Schiffman EL. Effect of Shortened Dental Arch on Temporomandibular Joint Intra-articular Disorders. J Oral Facial Pain Headache. 2018;32(3):329-37. doi:10.11607/ofph.1910

8. Okeson JP. Evolution of occlusion and temporomandibular disorder in orthodontics: Past, present, and future. Am J Orthod Dentofacial Orthop. 2015;147(5 Suppl):S216-S223. doi: 10.1016/j.ajodo.2015.02.007

9. Palma LF, Rocha PR, Chaddad Neto FEA, Smith RL, de Moraes LOC. Irrigation fluid volume requirement for conventional arthrocentesis of the temporomandibular joint: a cadaver study. Int J Oral Maxillofac Surg. 2020;S0901-5027(20)30103-X. doi: 10.1016/j.ijom.2020.03.012

10. McCain JP, Hossameldin RH. TMJ Arthroscopy. En: Tiwana PS, editor. Atlas of Oral and Maxillofacial Surgery. [Philadelphia]:Sauders; 2016. p. 1310-19.

11. Foletti JM, Cheynet F, Graillon N, Guyot L, Chossegros C. Arthroscopie de l'articulation temporo-mandibulaire. Mise au point [TMJ arthroscopy. A review]. Rev Stomatol Chir Maxillofac Chir Orale. 2016;117(4):273-79. doi: 10.1016/j. revsto.2016.07.010

12. Sidebottom A, Murakami A. Arthrocentesis and Arthroscopic. Management of the Temporomandibular Joint. En: Brennan PA, Schliephake H, Ghali GE, Cascarini L, editores. Maxillofacial Surgery. 3.a edición. [Philadelphia]: Elsevier; 2017. p. 147379.

13. Pastore GP, Goulart DR, Pastore PR, Prati AJ. Removal of a Solitary Synovial Chondromatosis of the Temporomandibular Joint Using Arthroscopy. J Craniofac Surg. 2016;27(4):967-69. doi:10.1097/SCS.0000000000002612

14. Holmlund A. Arthroscopy and Arthroscopic Surgery. En: Andersson L, Kahnberg KE, Pogrel A, editores. Oral and Maxillofacial Surgery. [Singapore]:Wiley; 2010. p. 1197-1208.

15. Foletti JM, Cheynet F, Graillon N, Guyot L, Chossegros C. Arthroscopie de l'articulation temporo-mandibulaire. Mise au point [TMJ arthroscopy. A review]. Rev Stomatol Chir Maxillofac Chir Orale. 2016;117(4):273--79. doi: 10.1016/j. revsto.2016.07.010

16. SroujiS, OrenD,ZoabiA, RonenO,ZraikH. Temporomandibular joint arthroscopy technique using a single working cannula. Int J Oral Maxillofac Surg. 2016;45(11):1490-94. doi: 10.1016/j. ijom.2016.05.016

17. Monje Gil F. Surgical management of temporomandibular joint. En: Arthroscopy. [Spain: iBooks-]; 2014. p. 1-154.

18. Murakami K, Clark G.T. Diagnosis of intracapsular pathology associated with temporomandibular joint disorders. Adv Dent Res. 1993;7(2):120-26.

19. Kaminishi RM, Davis CL. Temporomandibular joint arthroscopic observations of superior space adhesions. Oral Maxillofac Surg Clin North Am. 1989;1:93-102.

20. Carls FR, Engelke W, Locher MC: Complications following arthroscopy of the temporomandibular joint: analysis covering a 10-year period (451 arthroscopies). J Craniomaxillofac Surg. 1996;24:12-15.

21. Sato FRL, Lima CAA, Tralli G, da Silva RA. Is there a correlation between arthroscopic findings and the clinical signs and symptoms of patients with internal derangement of the temporomandibular joint? A prospective study. Int J Oral Maxillofac Surg. 2019;48(2):233-38. doi: 10.1016/j. ijom.2018.07.004

22. Holmlund A, Hellsing G. Arthroscopy of the temporomandibular joint. Occurrence and location of osteoarthrosis and synovitis in a patient material. Int J Oral Maxillofac Surg. 1988;17:36-40.

23. Dijkgraaf LC, Spijkervet FK, de Bont LG. Arthroscopic findings in osteoarthritic temporomandibular joints. J Oral Maxillofac Surg. 1999;57(3):255-68; discussion 269-70. doi: 10.1016/s0278-2391(99)90669-9

24. Wilkes $\mathrm{CH}$. Internal derangements of the temporomandibular joint. Pathological variations. Arch Otolaryngol Head Neck Surg. 1989;115(4):469-77. doi: 10.1001/ archotol.1989.01860280067019

25. Bronstein SL, Merrill RG. Clinical staging for TMJ internal derangement: Application to arthroscopy. J Craniomandib Disord Facial Oral Pain. 1992;6(1):7-16.

26. González-García R. The current role and the future of minimally invasive temporomandibular joint surgery. Oral Maxillofac Surg Clin N Am. 2015;27:69-84. doi: 10.1016/j. coms.2014.09.006.

27. Fernández Sanromán J, Costas López A, Fernández Ferro M, de Sánchez AL, Stavaru B, Arenaz Bua J. Complications of temporomandibular joint arthroscopy using two-portal coblation technologies: A prospective study of 475 procedures. J Craniomaxillofac Surg. 2016;44(9):1221-25. doi: 10.1016/j. jcms.2016.06.027

28. Chowdhury SKR, Saxena V, Rajkumar K, Shadamarshan RA. Complications of Diagnostic TMJ Arthroscopy: An Institutional Study. J Maxillofac Oral Surg. 2019;18(4):531-35. doi:10.1007/s12663-019-01202-3

29. Murakami K, Hosaka H, Moriya Y, Segami N, Iizuka T. Short-term treatment outcome study for the management of temporomandibular joint closed lock. A comparison of arthrocentesis to nonsurgical therapy and arthroscopic lysis and lavage. Oral Surg Oral Med Oral Pathol Oral Radiol Endod. 1995;80(3):253-7. doi: 10.1016/s1079-2104(05)80379-8

30. Avellá Vecino FJ, Salazar Fernández CI, Gallana Álvarez S, Rollón Mayordomo A, Mayorga Jiménez F, Pérez Sánchez JM. La artroscopia en el daño interior de la ATM: resultados clínicos de un estudio prospectivo. Rev Esp Cirug Oral y Maxilofac. 2004; 26(2):77-85.

31. Dahlström L, Widmark G, Carlsson SG. Changes in function and in pain-related and cognitive-behavioral variables after arthroscopy of temporomandibular joints. Eur J Oral Sci. 2000;108(1):14-21. doi: 10.1034/j.1600-0722.2000.00757.x

32. McCain J.P, Israel H, Choi D, Behrman D, Grewal N. Arthroscopy of Temporomandibular Joint Prosthesis, Preliminary Experience and Results. J Oral Maxillofac Sur. 2018,76(10): 32 . 